lead salt can enhance the catalytic oxidation of ethanol on platinum.

Ethanol can be used as an energy source for fuel cells and has several advantages over methanol, being less toxic and having a higher energy density. However, its complete oxidation to carbon dioxide on platinum, which requires the breakage of a $\mathrm{C}-\mathrm{C}$ bond, is stunted because of its preference for binding to the surface through its oxygen atom. This leads to unwanted stable products such as acetaldehyde and acetic acid that resist further oxidation.

Mukerjee and colleagues have now shown how the addition of $\mathrm{Pb}(\mathrm{IV})$ acetate to the reactant solution above a supported $\mathrm{Pt} / \mathrm{C}$ heterogeneous catalyst enhances the complete oxidation of ethanol. Improvements to the oxidation process were revealed by various electrochemical measurements, and showed a three-fold improvement in current density and far better stability compared with a platinum catalyst without the $\mathrm{Pb}(\mathrm{IV})$ acetate co-catalyst.

$\mathrm{X}$-ray absorption spectra, and their comparison with theory, suggest that when the co-catalyst is present the preferred adsorption geometry of the ethanol to the catalyst surface is through its methyl carbon, rather than oxygen. Mukerjee and colleagues suggest possible mechanisms by which the co-catalyst could cause this, one of which involves water-mediated non-covalent interactions between lead cations on the electrode surface and ethanol. These serve to orient the ethanol so as to encourage carboncatalyst interactions rather than those between its oxygen atom and the catalyst. $G A$

\section{ANION TOPOLOGY}

\section{Surprising sulfate species}

Angew. Chem. Int. Ed. http://doi.org/fz43ft (2012)

Sulfate anions $\left(\mathrm{SO}_{4}{ }^{2-}\right)$, which adopt a sulfur-centred tetrahedral form in which each apex is an oxygen atom, do not often condense into oligosulfates - and when they do they typically form chainlike compounds. Henning Höppe and co-workers at the Universities of Augsburg and Freiburg in Germany have now prepared an unusual polysulfate anion by reacting together boric acid, potassium sulfate and sulfuric acid. The uncommon topology of the crystalline potassium borosulfate obtained, $\mathrm{K}_{5}\left[\mathrm{~B}\left(\mathrm{SO}_{4}\right)_{4}\right]$, was revealed by characterization through single-crystal and powder X-ray diffraction, infrared and Raman spectroscopy, and supported by computational calculations.

In the potassium borosulfate crystal obtained, sulfate anions do not undergo direct condensation. Instead, four sulfate tetrahedra coordinate to a central boron atom - an unusual environment for boron - each of them through a bridging oxygen atom. The resulting borosulfate moieties are discrete ionic $\mathrm{B}\left(\mathrm{SO}_{4}\right)_{4}{ }_{4}$ - assemblies that crystallize with potassium counter-cations in a polar lattice.

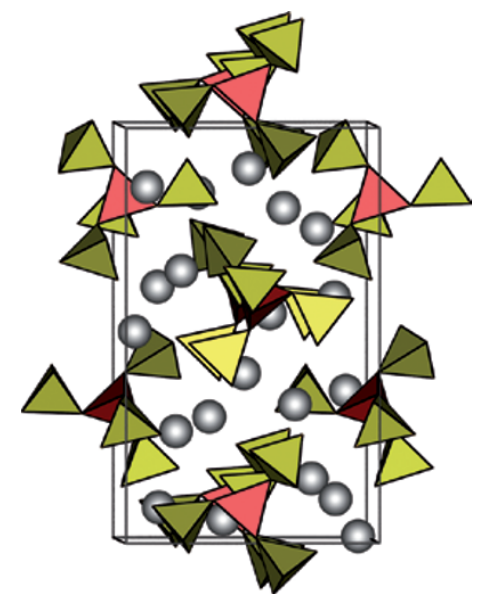

Such a topology has not been observed before with either sulfate or phosphate anions, which adopt the same tetrahedral morphology and have a similar size.

Furthermore, it is also rare in nature; it has only been described for the tetrahedral silicate anions $\left[\mathrm{Si}\left(\mathrm{SiO}_{4}\right)_{4}\right]^{12-}$ in the mineral zunyite. In that case, however, the silicate species also connect to aluminate octahedra $\left(\mathrm{AlO}_{6}\right)$ to form an extended network.

For each sulfate tetrahedron, the three terminal oxygen atoms - not involved in coordinating the borate atom - are slightly closer to the central sulfur atom than their bridging counterparts. This is consistent with a length discrepancy between bridging and terminal oxygen atoms previously observed for the disulfate anions of $\left[\mathrm{Si}\left(\mathrm{S}_{2} \mathrm{O}_{7}\right)_{3}\right]$.

The force constants calculated for both types of S-O bonds and for B-O bonds are also in good agreement with those found for the disulfate $\left(\mathrm{S}_{2} \mathrm{O}_{7}{ }^{2-}\right)$ and tetrahydroxyborate $\left[\mathrm{B}(\mathrm{OH})_{4}\right]^{-}$anions. The terminal oxygen atoms interact with the potassium counter-cations incorporated within the cavities of the borosulfate lattice. The electrostatic consistency of the structure of this borosulfate anion was supported by computational calculations.

Written by Gavin Armstrong, Stuart Cantrill, Stephen Davey and Anne Pichon.

\section{Correction}

In the print version of the Research Highlight 'Panning for gold' ( $\mathrm{H}$. Powell Nature Chem. 4, 431; 2012), the credit line for the image should have read: (C) ACS 2012.

\section{blogroll $^{\text {i }}$}

\section{\#WhatsInLemiShine}

A real-time collaborative determination of an unknown chemical compound, and a graduate student tackles teaching organometallic chemistry

Remember when Paul Docherty of Totally Synthetic (http://go.nature.com/c6c5ST) live-blogged an attempt to reproduce the reported oxidation of benzylic alcohols with sodium hydride? Adam Azman of Chemistry Blog recently went a step further in both speed and collaboration. During a conversation on Twitter about the identity of the trade-secret dishwasher detergent additive Lemi Shine and its remarkable ability to solubilize hardwater stains, Azman asked what chemists would like to know to identify the active ingredient. Answers from around the world flooded in, requesting both physical property data and instrumental analyses.

The following day, Azman performed wet chemistry to determine the identity of the compound, all while reporting his results on Twitter and taking suggestions for further experiments. Even images of GC-MS traces and NMR spectra ( ${ }^{1} \mathrm{H}$ and ${ }^{13} \mathrm{C}$ ) were posted. The conclusion? Lemi Shine's active ingredient was citric acid. When asked for comment from Lemi Shine (via Twitter), they responded with a cryptic "Oh so close, but no cigar." To see if you agree with Azman as to the identity of the active ingredient, go to Chemistry Blog for a summary of the experiments (http://go.nature.com/SmF9MX). This episode has also inspired others to tweet experimental chemistry results, all under the hashtag \#RealTimeChem.

How are you on the principles of backbonding? Forgotten the details of an open coordination site? Graduate student Michael Evans says that "there is a better way to teach organometallic chemistry." Towards that end, he's started The Organometallic Reader (http://go.nature. com/xV29ce), where he posts an in-depth lesson each week. These excellent, readable posts are a good primer for the novice and a nice refresher for the experienced chemist.

Written by Chemjobber who blogs at http://www.chemjobber.com 